\title{
Conf $770383-4.7$
}

SUPERCONDUCTING HEAVY-ION LINACS

Lowe11 M. Bollinger

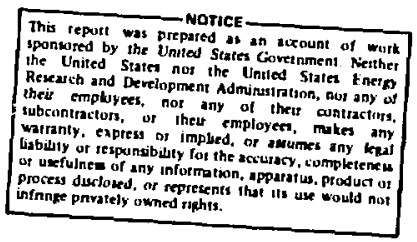

Prepared for

Particle Accelerator Conference

Chicago, Illino is

March 16-18, 1977
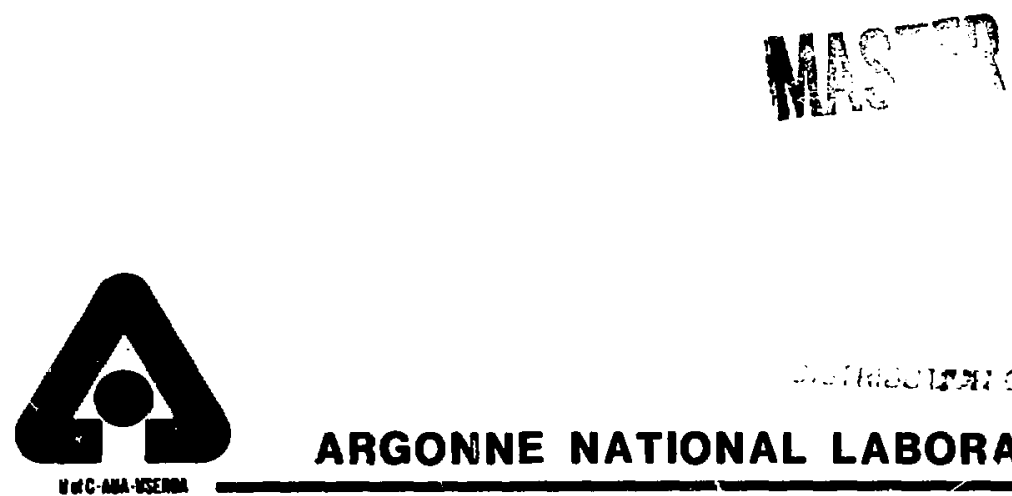

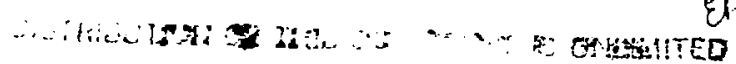

ARGONNE NATIONAL LABORATORY, ARGONNE, ILLINOIS

operated under contract W-31-109-Eng-38 for the

U. S. ENERGY RESEARCH AND DEVELOPMENT ADMINISTRATION 


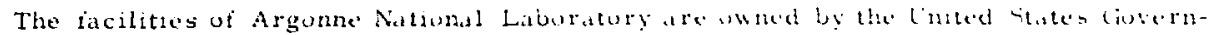

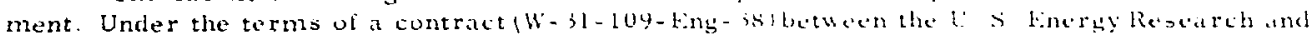

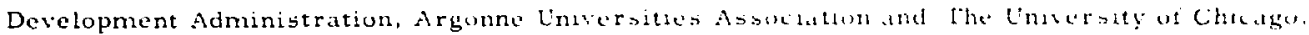
the University empluys the stait and operates the Laburatory an acoudane with policies and

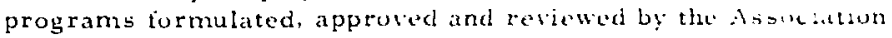

\section{MEALERS OF ARGONE LNWHSIMS ASSOCATION}

The University of Arizona

Carnegie-Mellon University

Case Western Reserve Unwersity

The University of Chicago

University of Cincinnati

Illinois Institute of Technology

University of Illinois

Indiana University

lowa State University

The University of Iowa
Katnsils State Linuersity The Lutersity of Kansas Loyolat Unaresity

Marquette Lnaverasty Mublatalu State Lnacersty The Lnuersity of Mtichledn Unversoly at Monuesota Unversaty ut Massuri Northwestern Lnaversity Unutersity at Wotre Dame
The Ohio State Unurersity

Ohas Crumersty

The Pennsylinala State Unversity

Purdue Lnaversity

Sam Inum Untersity

Southern lllinois Liniversity

The University of lexas at Austin

Washington University

Wayne State University

The University of Wisconsin

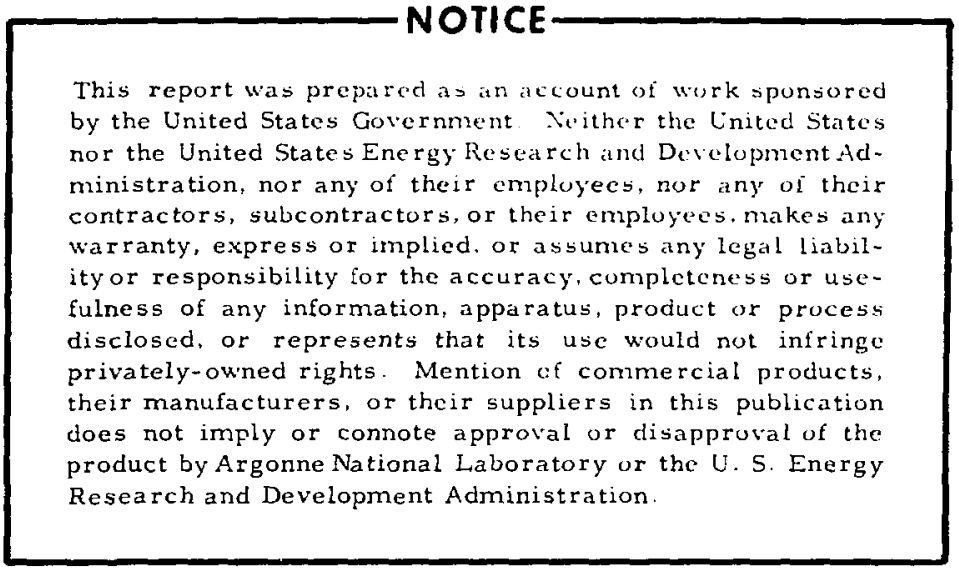


SUPERCONDUCTING HEAVY-ION LINACS

Lowell M. Bollinger

Argonne National Laboratory

Argonne, Illinois 60439

To be Published in the Proceedings

of the

1977 Particle Accelerator Conference

March 16-18, 1977

Chicago, Illinois 
Lowell M. Bollinuer

Argonne National Laboratory

Árgonne, Jllinois 60439

The paper summarizes plans developed by four different groups for the construction of small suparconducting 1 inacs to boost the energy of heavy ions from existing tandem electrostatic accelerators. The projects considered are the linac under construction at Argonne and the design efforts at Karlsruhe, at Stanford, and by a Cal Tech-Stony Brook collaboration. The intended uses of the accelerator systems are stated. Beam dynamics of linacs formed of short independently-phased resonators are reviewed, and the implications for performance are discussed. The main parameters of the four 1 inacs are compared, and a brief analysis of accelerating structures is given.

\section{Introduction}

The aim of this paper is to review four projects concerned with the design and construction of small superconduct ing 1 inacs for heavy-ion acceleration. These are the projects at Argonne 1, a Cal Tech-Stony Brook collaboration ${ }^{2}$, a proposal by a Karlsruhe ${ }^{3}$ group to build a linac at Heidelberg, and the Stanford 4 project. of these, only the Argonne linac is under construction; the others are all in the stage of proposals based on the results of extensive developmental efforts. This paper outlines the main features of the four design concepts.

All of the linacs considered are to serve as boosters of heavy ions from existing tandem electrostatic accelerators. The main components of a representative tandem-linac system are outlined in Fig. 1. One starts with a negative-ion source at the low-energy end of the tandem. The bean from the source is partially bunched before injection into the tanden.

After acceleration to the tandem terminal, the negative ions are stripped in either a foil or in gas, and the multiply-charged positive ions so formed are accelerated back to ground potential.

After passing through an analyzing magnet, where a single charge state is selected, the bean is further bunched by high-field resonator in order to produce the narrow pulses required for optimum acceleration in the 1 inac. Also, either before or after the tanden, unbunched particles are removed by means of a chopper.

Because of the long flight path, the time required for an ion to pass from the pretanden buncher to the post-tandem buncher is unsteady. Consequently, sone means is required to detect the rf phase with which ion bunches arrive at the post-tandem buncher. This phase signal controls the phase of the pre-tandem buncher. Before being injected into the linac, the ion bean is stripped a second time. If the Work performed under the auspices of the U.S. Energy Research ant Devolopaent Administration required charge-state selector is located after the linac, then several charge states may be accelerated through the linac.

In all projects considered, the linac itself is an array of short independently-phased resonators. Interspersed with these are focusing elements (quadrupoles or solenoids) that 1 imit the beam size.

The superconducting linacs are being justified, to some extent, as a contribution to the development of a new technology. lowever, there is also a keen interest in using them for research. All of the accelerator systems are aimed at the needs of nuclearstructure research. The energy of most interest for such, research is the region near the Coulomb harrier, that is, from a few MeV per nuclenn to perhaps $15 \mathrm{MeV}$ per nucleon. The accelerator systems consiclered here will effectively extend rescarch into this hind for ions in the lower part of the periodic table but leave untouched the high-mass region.

An accelerator for nuclear-structure research should have easy energy variability and it should provide ion beams of very good qual. ity both with respect to transverse and longitudinal emittance. These characteristics are natural attributes of the tandem-1inac systen.

\section{Beam Dynanics}

An important feature of the linacs considered is that they are formed of shoit independent $1 y$-phased resonators. The essential difference between then and a conventiona) linac is that the velocity profile is established by phasing rather than by the increasing lengths of successive accelerating units. Since the velocity need not be matched to the resonator length, the projectile phase may change greatly while it truverses the structure. Nevertheless, phase focusing is present in the same way as in a long structure with a well-established velocity profile. Consequently, the internal behavior of an ion

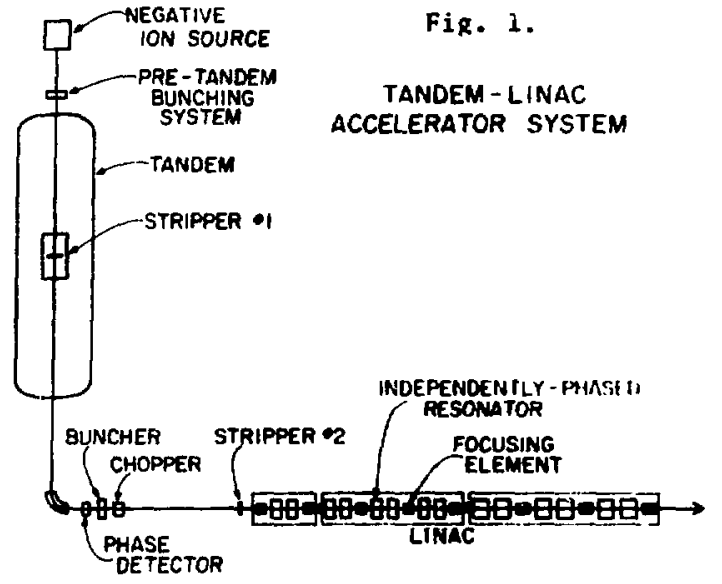




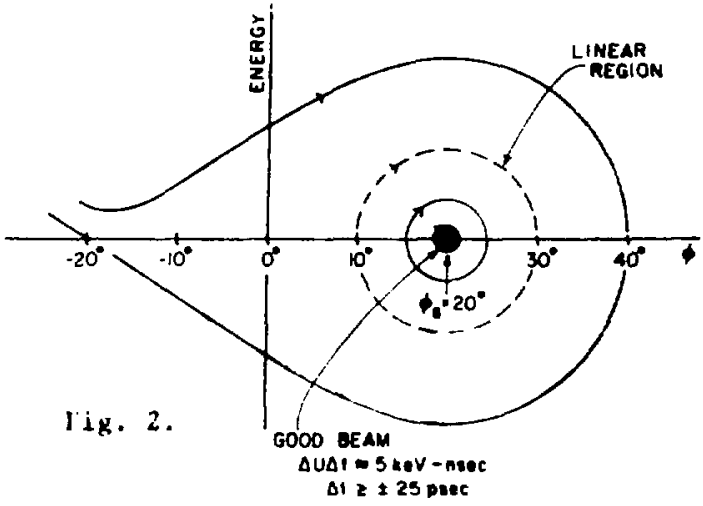

bunch can be described 5 in terms of an equivalent phase space, as shown in Fig. 2 , where the acceleration process is treated as though the center of the bunch were synchronous with the forward-travelling wave in a conventional long structure. Here $\Delta U$ is the deviation from the average energy of the bunch, and the synchronous phase is is the average phase of bunch in each resonator. Within the circle marked "linear region", the phase-restoring forces are accurately proportjonal to $\phi_{s}-\phi$ and, if the beam can be confined to this region, bean quality is preserved.

Sometimes it may be advantageous to shape and place the bunch in phase space so as to emphasize a specific beam characteristic at the linac output. Such flexibility requires a sophisticated bunching system.

A short independently-phased resonator can effectively accelerate a wide range of velocities. This is illustrated in Fig. 3, where the transit-time factor (defined by the equation on the figure) is given as a function of $\mathrm{U} / \mathrm{U}_{\mathrm{S}}$ for two structures, where $\mathrm{U}_{\mathrm{s}}$ is the synchronous energy of the structure. A linac formed of such resonators is exceedingly flexible with regard to the mode of operation and hence is tolerant of sub-standard performance of resonators. A failure of resonators to provide the design accelerating field will reduce the maximum beam energy, but the 1 inac can continue to function usefully.

The transit-time factor of a single resonator does not give a direct indication of how transit-time effects influence the output energy of an accelerator system. Rather, one

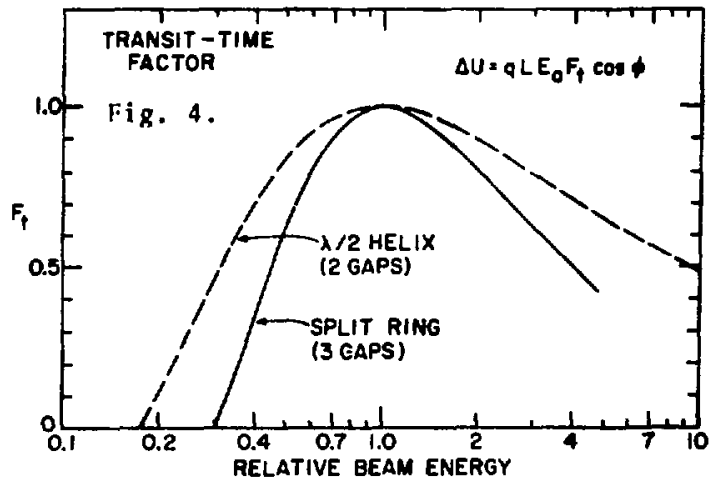

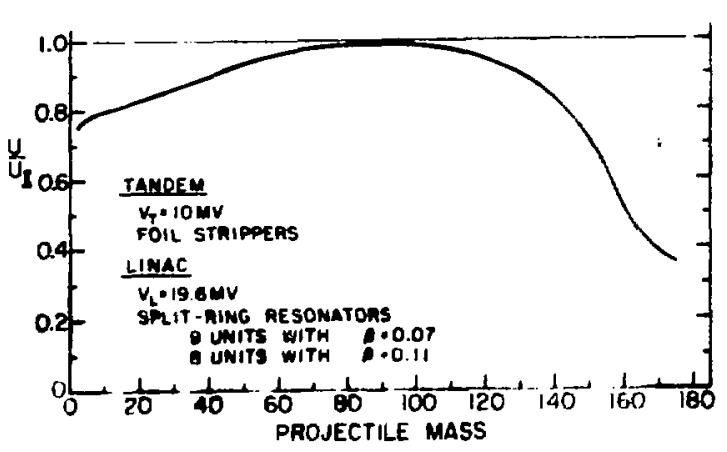

Fig. 4. Accelerating efficiency

needs to know, for a particular system, the mass dependence of the ratio $U / U_{I}$, where $U$ is the output energy and $U_{I}$ is the energy of an idealized system in which the transit-time factor is unity for all resonetors. An

exanple is given in Fig. 4. Even though only two sizes of resonators are used, the linac accelerates efficiently over wide range of mass.

\section{Bunching}

A sophisticated bunching system is required for an opt imum tandem-1inac accelerator. One important requirement is to match the longitudinal phase space of the beam to the 1 inac. As indicated in Fig. 2, one may wish to have ion bunches as narrow as \pm 25 psec, which is almost two order of magnitude narrower than is now 5 tandard practice. Moreover, because of low beam currents for some ions, the buncher should bunch a large fraction (say $80 \%$ ) of the dc beam from the source. Calculations indicate that these requirements can be met by bunching in two stages, first before the tandem and then after the tandem. Of these, the first stage is by far the most difficult since there the ions are moving extremely slowly, and several time-spreading effects are impor tant. Post-tandem bunching to form ultranarrow pulses has been shown experimental1 $y^{6}$ to be easy.

The accelerator user may require a bunching frequency that ranges from perhaps $5 \mathrm{MHz}$ up to the rf frequency. This challenge has not been accepted yet by buncher designers.

\section{Accelerating Structures}

The accelerating structures chosen for use are illustrated in Fig. 5, a scale drawing of the inner dimensions of resonators tested to date. Let us briefly consider the main features of each design.

The Argonne split ring 7.8 is a large structure (for a cryogenic system) designed for the high-energy end of the linac. A lowbeta unit is under development. The rf frequency is a compromise between a desire to have as low a frequency as possible (to minimize the bunching problem and to maximize the accelerating length of an individual unit), on the one hand, and the desire to 1 imit the radial dimensions and stored energy, on the other. The split-ring tube is shaped so as to 

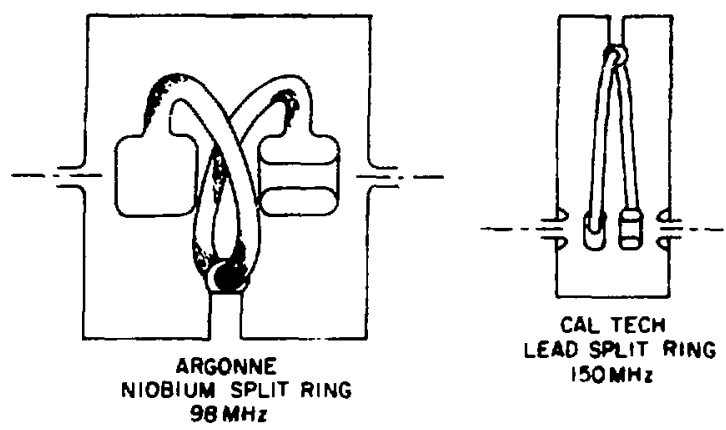

CAL TECH

LEAD SPLIT RING $150 \mathrm{MHz}$

$98 \mathrm{MHZ}$
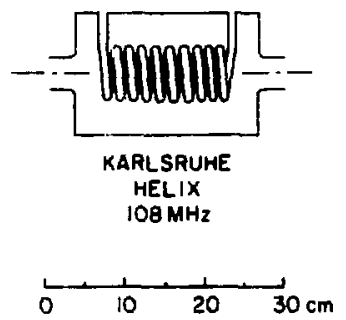

Fig. 5

make the drift tubes coaxial with the housing, thus minimizing the radial size. The structure is mechanically stiff, and hence the $r f$ frequency is insensitive to mechanical vibrations and radiation pressure. The drift tubes are large in order to minimize surface electric fields, sensitivity to mechanical misalignments, and beam-quality deterioration. These desirable features are obtained at the cost of increased stored energy, which increases power dissipation and magnifies the phase-control problem.

The Cal Tech split ring 2,9 tested to date is designed for the low-energy end of the linac. A high-beta unit is under development. The superconductor is lead on copper, which is attractive from the point of view of ease of fabrication and cryogenic simplicity. Because of the high $r f$ frequency (relative to the Argonne design), the radial size is acceptable even though the drift tubes are not coaxial with the housing. The $Q$ of the structure is lower than that of most superconducting structures because the surface resistivity of lead is higher than that of niobium.

The Karlsruhe $\lambda / 2$ helix ${ }^{3 / 10}$ is a structure with $B=0.09$ designed for use throughout the linac. A special design feature is that, to reduce fabrication costs, the resonator is formed entirely by arc welding (rather than beam welding). Also, the end plates are welded on rather than attached by means of demountable joints. Relatively large beam ports allow surface treatment to be carried out after the end plates are attached. The $1-\mathrm{cm}$ diameter of the helix wire is thick enough to make the helix fairly stiff. Nevertheless, phase control is still more difficult for the helix than for the other structures. Large end effects of the helix structure limit the average energy gain per unit length achievable with $\lambda / 2$ units.
The Stanford re-entry cavity Il $^{\text {is based }}$ on the technology developed for the stanford superconducting electron linac. The rood features of the design are a) the axia! innmetry, which eliminates beam-steering effects that are present to come extent in the other resonators, h) the vide velocity acceptance of a single-gap structure, and c) mechanical rigidity. These advantages are obtained at the cost of an exceptionally strong sensitivity to multipacting (because of spacial symmetries). a low average field fradient, and in uncomfortably high rf frequency.

Ref. $2-4,7-11$ pive details on resonator performance. Some features common to all are out Iined in Fip. f. llere, for a representative unloaded structure, $\subseteq$ is plotted as a function of the maximum surface electric field Esuly. In the low-field region, the loss results mainly from the surface resistivity of the superconductor, whereas in the high-field region, electron field emission is dominant.

The maximum field strength that can be achieved is often detcrmined by conling. A second set of curves in Fig. 6 give $Q$ vs Es for a fixed level of power dissipation. These curves are approximately valid for either a split-ring or a helix structure at $100 \mathrm{MHz}$; and a power loss in the neighborhood of 25 Watts per meter of active length is representative of the planned linacs.

The point of intersection of appropriate power-loss and resonator-performance curves gives the maximum operating field of a structure. In practice, for the low-frequency niobium structures, at least, electron field emission usually sets the operating field.

\section{Cryogenics and Phase Control}

In order to minimize costs, all groups have decided to operate the resonators at temperatures ncar $4.5^{\circ} \mathrm{K}$. Recent experience with low-frequency structures has shown that there is no significant temperature dependence of performance for $\mathrm{T}<4.5^{\circ} \mathrm{K}$. Apparent $1 \mathrm{y}$, the residual resistivity caused by surface defects

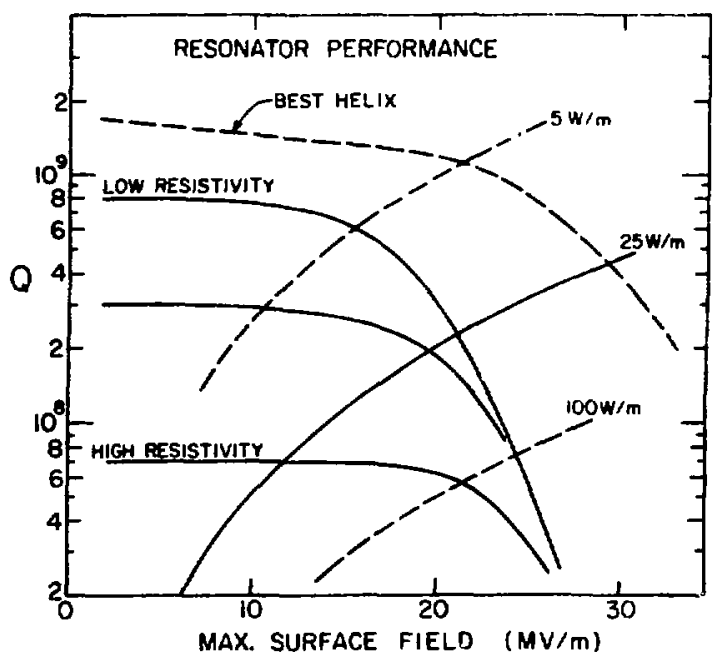

Fig. 6. Resonator performance. 
in a practical superconductor are dominant and insensitive to temperature.

The extremely narrow band width of a superconduct ing resonator and the larfe ize of a structure suitable for heavy ions cause frequency varjations to be important and make phase control a significant problem. However, effective means of control have been found for all of the structures considered here.

There are two main elements of a practical phase-control system: 1) an accelerating structure that is rigid enough to limit mechanical motion, and 2) some form of electrical feedback. Perhaps the most elegant electrical control is that used with the Cal Tech split ring, namely, negative phase feedback. The much higher stored energy of the Argonne split ring and the larger frequency variation of the Karlsruhe helix would make phase feedback an expensive approach; instead, both are contolled by a voltage-controlied reactance (VCX) that makes use of reactive power and involves switching PIN diodes.
The Stanford approach is to use a combination of mechanical deformation of the cavity wall and negative feedback. This technique has not been tried on less rigid structures hecause of fear that the controlling motion may induce harmful vibration.

\section{Summary of Jinac Parameters}

Important parameters of the four linacs are summarized in Table 1. The best simple measure of a linac's accelerating poser is its total voltage gain $V_{L}$, which gives a rough indication of the output energy $U$ frem the relationship

$$
\mathrm{U} \leqslant \mathrm{u}_{\mathbf{O}}+\mathbf{q} \mathbf{v}_{\mathbf{L}} \text {. }
$$

wherc $v_{0}$ is the incident energy and $q$ is charge. Fig. 4 gives an example of the accuracy of the equation, since $\mathrm{U}_{0}+q \mathrm{~V}_{\mathrm{I}} \equiv \mathrm{U}_{\mathrm{I}}$. A $20-\mathrm{MV}$ 1 inac injected by a smail tandem is approximately equivalent to a $25-M N$ tandem for ions in the lower third of the periodic table.

TABLE 1. Linac Characteristics

\section{Linac Size}

Overall length (meters)

Active length (meters)

\section{Resonators}

Type

Superconducting material

RF frequency (MHz)

Number and design $B$ :

Design objectives:

Max. surface $F(\mathrm{MN} / \mathrm{m})$

Max. surface $B(m T)$

Minimum Q

\section{Cryogenics}

Operating temperature $\left({ }^{\circ} \mathrm{K}\right)$

Total refrigeration (Watts)

Mode of helium cooling

RF Power and Controls

Total RF power (kw)

Mode of phase control

Transverse Focussing

Type of element

Number of elements

Bunching

Pre-tandem

Post-tandem

Injector

Type of tandem

Max. voltage (MV)

Performance

Design objective:

Max. voltage gain (MV)

Ion with $U_{\max }=5 \mathrm{MeV} / \mathrm{A}$

worst credible performance:

Max. voltage gain (MV)

Ion with $U_{\max }=5 \mathrm{MeV} / \mathrm{A}$

Scatu:
Cal Tech.

Stony Brook

$\frac{\text { Argonne }}{9.6} \cdot \frac{\text { Stony Brook }}{4.7}$
split ring
lead

split ring

niobium

98

12, $B=0.105$

20

67

$4 \times 10^{8}$

4.7

flowing $2-\phi$

$$
2.8
$$

vCX

SC solenoid

8

yes

yes

FN-mode 1

9. 5

20

75

12

60

under

construction

16, $B=0.055$

18

33

$1 \times 10^{9}$

4.2

pool boiling

10

RT Quad

12

yes

FN-mode 1

9.5

20

90

13

65

advanced design

$\frac{\text { Karlsruhe }}{19} \quad \frac{\text { Stanford }}{12}$

$\begin{array}{cc}\lambda / 2 \text { helix } & \text { reentrant } \\ \text { niobium } & \text { niobium } \\ 108.5 & 430 \\ 40, B=0.095 & 90, B \leq 0 .\end{array}$

- $\phi$ feedback

$\stackrel{4}{\text { VCX }}$

0.6

12

18

62

$5 \times 10^{7}$

$5 \times 10^{8}$

4.5

200

flowing $2-\phi$

4.2

200

bath

Piezo-El.

SC solenoid

10

SC. solenoid

yes

yes

no

yes

MP-mode 1

13

FN-mode 1

8.5

10

63

1.

9
50

14 advanced

design
37

advanced design 


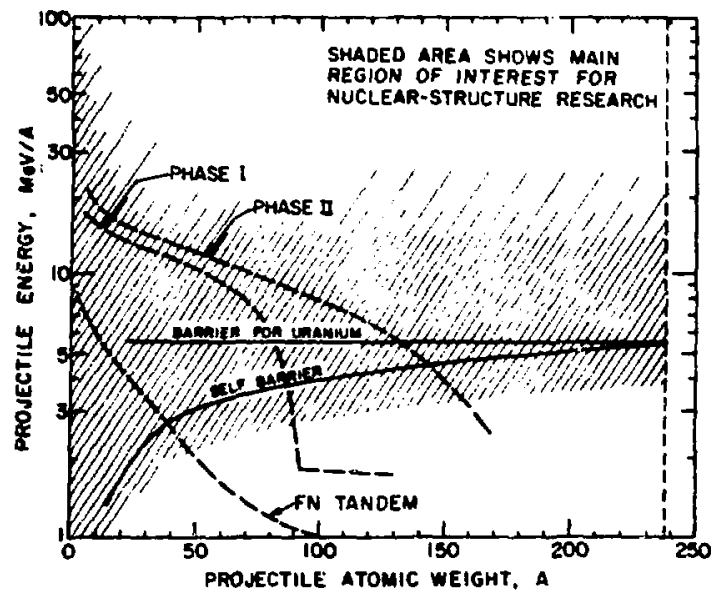

Fig. 7. Representative performance.

The inverse relationship between the effective length of an accelerating structure and the number of units required to form a linac is apparent in the table. The optimum design is a compromise between having a minimum number of resonators and having individual units that are small enough to be manageable. More experience is required before this optimum is located with certainty.

Refrigerator sizes are influenced greatly by'economics. However, a larger refrigerator would not increase the operating field greatly because field emission increases so rapidly with increasing field.

The total of power required is small for all of the linacs under discussion, and most of it is used for phase control.

\section{Per formance}

Bean Energy

The accelerating powers of the systems under discussion are specified in Table 1 . More detail is given in Fig. 7 for one system (Argonne), where the Phase I curve gives energy performance that is representative of a 20-MV Iinac formed largely of resonators of one size. The Phase 11 curve shows how the performance can be extended by the addition of only 6 low-B resonators.

\section{Energy Variability}

The output energy can be varied over a considerable range merely by changing the phase of the last resonator of the linac. If the phase is restricted to the almost linear part of the acceleration curve, then the output phase ellipse is almost independent of phase. A wider energy range can be covered by turning off resonators.

Beam Current

The Iinac is expected to accelerate almost all ions injected into it if they have the same charge. Thus, the output beam is determined almost entirely by the tandem and by stripping. If two strippers are used, the number of ions out of the linac can be about $2 *$ of the number injected into the tandem. Typically, the output will be 1011 ions per sec, which is adequate for most nuclearstructure research, especially in view of the good healn quality.

Beam Quality If beam bunching is refined enough, the ion beam incident on the linac can be acselerated without a significant deterioration of beam quality. Thus, beam quality is estab. 1 ished by the tander and by bunching and stripping. Typically, the transverse enittance of the 1 inac output is expected to range from 1 to $10 \mathrm{~mm} m \mathrm{mad}$, depending on the ion and the strippers.

The longitudinal emittance (the product cust) is determined primarily by pre-tandem bunching and stripping. The optimum is a system in wich the post-tandem buncher forms a time focus at the second stripper so as to minimize the inflisence of straggling. Then $\Delta U \Delta t$ is expected to be in the range 5 to 50 $\mathrm{keV}$ nsec. depending on the ion and on the stripper quality. These values correspond to a convention in which both $\Delta U$ and $\Delta t$ are half widths at half maximun of the distributions.

The energy and time spreads of the linacoutput beam depend on many operating parameters. Typically, however, an incident beam of good quality ( $\Delta U \Delta \Delta t=5 \mathrm{keV}$ nsec) in the Argonne linac gives $\Delta U / U= \pm 0.5 \times 10^{-3}$ and $\Delta t= \pm 25$ psec.

If the experimenter requires either better energy resolution or hetter $t$ ime resolution, then the natural way to obtain it is to debunch or rebunch the beam, respectively. Typically, a debuncher-rebuncher system requires just one conventional resonator, and a flight path about 10 meters long can improve the energy resolution by a factor of 5 . The time resolution can also be improved by a comparable factor, if the beam pulse is not extremely narrow initially.

\section{References}

1. L. 4 . Bollinger et al, in Proc. of the 1976 Proton Linear Accelerator conf. AECL-5677, p. 95 (1976).

2. J. W. Noé et al., this conference.

3. 6. Hochschild et al., this conference.

4. J. S. Sokolowski et al., this conference.

5. T. K. Khoe, privatc communication (1976).

6. L. M. Bollinger et al. IEEE Trans. Nucl. Sci. NS-22, 1148 (1975).

7. R. Benaroya et a1,. IEEE Trans. Magnetics MAG-13, 516 (T977).

8. K. W. Shepard et al., this conference.

9. G. J. Dick et a1., Nucl. Instr. Methods, $138,203(1976)$.

10. J. E. Vetter et al., in Proc, of tne 1976 Proton Linear Accelerator Conf., AECL- 5677 p. 106 (1976).

11. P. H. Ceperley et al., IEEE Trans. Magnet ics $M A A-13,520$ (1977).

12. J. R. Delayen et al., this conference. 